\title{
AMOSTRAGEM DE CIGARRINHAS (HEMIPTERA, CICADELLIDAE) ATRAVÉS DE ARMADILHAS DE MOERICKE EM CAFEEIRO ARÁBICA
}

\section{R.I.R. Lara ${ }^{1}$, N.W. Perioto ${ }^{1}$, S. de Freitas ${ }^{2}$}

1Pólo Regional de Desenvolvimento Tecnológico dos Agronegócios do Centro Leste, Rua Peru, 1472-A, CEP 14075-310, Ribeirão Preto, SP, Brasil. E-mail: rirlara@aptaregional.sp.gov.br

RESUMO

Este estudo teve por objetivo avaliar o padrão de variação populacional de cigarrinhas (Hemiptera, Cicadellidae) associadas ao cafeeiro, em Cravinhos, SP. As amostragens foram realizadas, semanalmente, no período de abril de 2005 a abril de 2006 através de 60 armadilhas de Moericke instaladas nos terços médio e inferior das plantas. Foram coletados 3.243 exemplares de cigarrinhas pertencentes a 8 espécies: Bahita infuscata, Coelidiana diminuta, Dilobopterus costalimai, Docalidia bifurcata, Joruma (Joruma) sp., Labocurtidia sp., Oncometopia facialis e Scaphytopius irrorellus. As maiores abundâncias destes cicadelídeos associados à cultura do cafeeiro ocorreram no período chuvoso; as espécies de cicadelídeos coletadas foram constantes e as armadilhas de Moericke instaladas no terço médio das plantas foram mais eficientes para a captura deste grupo de insetos. Verificou-se correlação positiva significativa entre os valores populacionais de D. costalimai com a pluviosidade e as temperaturas máxima e mínima e, para $O$. facialis, foi apenas com a temperatura mínima.

PALAVRAS-CHAVE: Cigarrinhas vetoras, Coffea arabica, diversidade, flutuação populacional, Xylella fastidiosa.

\section{ABSTRACT}

SAMPLINGOF LEAFHOPPERS(HEMIPTERA, CICADELLIDAE) BYUSINGMOERICKETRAP IN COFFEE CROP. This study had for objective to evaluate the population fluctuations of the leafhoppers (Hemiptera, Cicadellidae) associated to Coffea arabica in Cravinhos, São Paulo, Brazil. The leafhoppers were sampled weekly by using 60 Moericke trap in the period of April/2005 to April/2006. Were collected 3.243 leafhoppers belonging to eight species: Bahita infuscata, Coelidiana diminuta, Dilobopterus costalimai, Docalidia bifurcata, Joruma (Joruma) sp., Labocurtidia sp., Oncometopia facialis and Scaphytopius irrorellus. The mostly frequency of the leafhoppers associated at coffee crop occurred in the rainy season; all the species of Cicadellidae collected were constants and the Moericke traps located at the median third of the plants were preferred for the capture of this group of insects. D. costalimai presented positive and significant correlations $(\mathrm{p}<0.05)$ with the rainfall and maximum and minimum temperatures and $O$. fascialis was only with minimum temperature.

KEY WORDS: Coffea arabica, diversity, population fluctuation, vectors leafhoppers, Xylella fastidiosa.

\section{INTRODUÇÃO}

O café é uma importante fonte de divisas para o BrasileoEstadodeSãoPaulodestaca-senoagronegócio desta mercadoria por abrigar o maior parque industrial de café do país e por sua infra-estrutura portuária, fundamental no escoamento da produção de outras regiões produtoras. A cultura de café Coffea arabica L. abriga inúmeras espécies de insetos e ácaros e, dentre estas, algumas se constituem em pragas importantes e podem causar prejuízos econômicos.
No Brasil, algumas espécies de cigarrinhas das famílias Cercopidae e Cicadellidae (Cicadellinae) transmitem a bactéria Xylella fastidiosa (LOPEs, 1996), agente causal da "atrofia dos ramos do cafeeiro" (PARAdela FilHo et al., 1997) cujos sintomas externos são entrenós mais curtos, diminuição no comprimento dos pecíolos e da área foliar, senescência das folhas mais maduras e flores e frutos agrupados e de tamanho reduzido (PARADELA FILHO et al., 1997; QUEIROZVOLTAN et al., 1998). As espécies de cigarrinhas que transmitem a bactéria $X$. fastidiosa podem ocorrer

${ }^{2}$ Universidade Estadual Paulista, Faculdade de Ciências Agrárias e Veterinárias, Departamento de Fitossanidade, Jaboticabal, SP, Brasil. 
também em plantas invasoras e em matas, podendo haver uma inter-relação entre esses locais de refúgio e alimentação (YАMAMOTO, 1998).

PARAdELA FilHo et al. (1997) relataram que as espécies de cigarrinhas Acrogonia terminalis Young, Dilobopterus costalimai Young e Oncometopia facialis (Signoret) são, freqüentemente, encontradas alimentando-se em plantas de café. Meneguim et al. (2000) acrescentaram àquelas espécies Sonesimia grossa (Signoret), Plesiommata corniculata Young, Homalodisca ignorata Melichar, Macugonalia leucomelas (Walker), Ferrariana trivittata (Signoret) e Bucephalogonia xanthophis (Berg.). Dada a importância desses insetos na transmissão do patógeno, este estudo teve por objetivo avaliar a diversidade e freqüência das espécies de cigarrinhas coletadas com armadilhas de Moericke na cultura do cafeeiro em Cravinhos, SP.

\section{MATERIALEMÉTODOS}

Oexperimento foi desenvolvidoem um cafezal da cultivar Obatã, de quatro anos de idade, plantado no espaçamento de $4 \times 1 \mathrm{~m}$, conduzido em sistema convencional e localizado na Fazenda Palmares

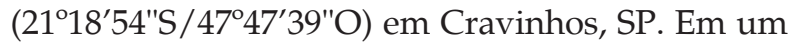
talhão de um hectare foram estabelecidos 20 pontos de amostragem e, em cada ponto, foram instalados três conjuntos de armadilhas de Moericke, perfazendo um total de 60 armadilhas, que permaneceram ativas em campo por $48 \mathrm{~h} /$ semana. Cada conjunto constituiu-se de dois pratos plásticos de coloração amarela ( $15 \mathrm{~cm}$ de diâmetroe $4,5 \mathrm{~cm}$ dealtura) fixados em estacas de madeira com auxílio de aros de arame conforme proposto por PERIOTO et al. (2000) de forma que a suas bordas ficaram na altura dos terços inferior emédio da planta (aproximadamente $0,4 \mathrm{me} \mathrm{1,0 \textrm {mem }}$ relação ao nível do solo); como conservante utilizouse solução de formol a 10\%; gotas dedetergenteneutro foram adicionadas à solução para quebrar a tensão superficial dolíquido. As amostragens foram realiza- das semanalmente, no período de abril de 2005 a abril de 2006.

A constância foi calculada segundo SILVEIRA-NeTO et al. (1976) e as espécies classificadas como constantes $(\mathrm{C}>50 \%)$, acessórias $(25 \%<\mathrm{C}<50 \%)$ e acidentais (C $<25 \%$ ) segundo Bodenheimer (1955) apud SILVEIRANeTo et al.(1976).

Oíndice de correlação de Pearson (r) foi utilizado para estabelecer possível relação entre a abundância semanal dos cicadelídeos, a pluviosidade e as temperaturas máxima e mínima observadas na semana anterior às coletas. Os dados meteorológicos foram cedidos pelo Escritório de Desenvolvimento Ruralde RibeirãoPreto(CATI), órgão da Secretaria de Agricultura e Abastecimento do Estado de São Paulo. Para o índice de correlação foi utilizado o software SAS.

Os exemplares estudados serão depositados na Coleção Entomológica de Parasitóides e Predadores (LRRP) e na Coleção do Museu Nacional do Rio de Janeiro(MNRJ).

\section{RESULTADOSEDISCUSSÃO}

Foram amostrados 3.243 exemplares de cicadelídeos pertencentes a cinco subfamílias, sete tribos e oito espécies (Tabela 1).

A abundância relativa das subfamílias de cicadelídeos coletadas no cafeeiro foi a seguinte: $48,5 \%$ para Coelidiinae(2 espécies / 1.574 indivíduos),32,0\% para Deltocephalinae (2/1040), 8,7\% para Cicadellinae (2/282), 5,8\% para Typhlocybinae (1/ 187) e $4,9 \%$ para Neocoelidiinae $(1 / 160)$.

Da subfamília Coelidiinae foram capturadas as espécies $D$. bifurcata e Labocurtidia sp., a última a mais abundante (43\%). A biologia dos membros desta subfamíliaé pouco conhecida: apenas Calodia kirkaldyi Nielson e Jikradia olitoria olitoria (Say) são relatadas como vetoras de vírus na Índia e nos Estados Unidos, respectivamente (Nielson, 1975, 1982) apud GoDOY \& NiELSON (1998).

Tabela 1 - Total de exemplares (N), freqüência (f \%), subfamílias, tribos e espécies de cigarrinhas coletadas com armadilhas de Moericke em cultivo de Coffea arabica, em Cravinhos, SP, Brasil. Período de abril de 2005 a abril de 2006.

\begin{tabular}{|c|c|c|c|c|}
\hline Subfamília & Tribo & Espécie & $\mathrm{N}$ & $f(\%)$ \\
\hline \multirow[t]{2}{*}{ Cicadellinae } & Cicadellini & Dilobopterus costalimai Young, 1977 & 88 & 2,7 \\
\hline & Proconiini & Oncometopia facialis (Signoret, 1854) & 194 & 6,0 \\
\hline \multirow[t]{2}{*}{ Coelidiinae } & Teruliini & Docalidia bifurcata Nielson, 1979 & 178 & 5,5 \\
\hline & & Labocurtidia sp. & 1.396 & 43,0 \\
\hline \multirow[t]{2}{*}{ Deltocephalinae } & Euscelini & Bahita infuscata (Osborn, 1923) & 419 & 12,9 \\
\hline & Scaphytopiini & Scaphytopius irrorellus (DeLong, 1944) & 621 & 19,1 \\
\hline Neocoelidiinae & Neocoelidiini & Coelidiana diminuta Chiamolera \& Cavichioli, 2005 & 160 & 4,9 \\
\hline Typhlocybinae & Jorumini & Joruma (Joruma) sp. & 187 & 5,8 \\
\hline Total & & & 3.243 & 100,0 \\
\hline
\end{tabular}


Tabela 2 - Abundância mensal e constância de cicadelídeos (Hemiptera, Cicadellidae) associados a Coffea arabica, em Cravinhos, SP, Brasil. Período de abril de 2005 a abril de 2006.

\begin{tabular}{|c|c|c|c|c|c|c|c|c|c|c|c|c|c|c|c|}
\hline \multirow[t]{2}{*}{ Cicadellidae } & \multicolumn{14}{|c|}{ número de cicadelídeos capturados } & \multirow{2}{*}{ Constância } \\
\hline & abr & mai & jun & jul & ago & set & out & nov & dez & jan & fev & mar & abr & total & \\
\hline Scaphytopius irrorellus & 31 & 69 & 6 & 39 & 34 & 22 & 7 & 13 & 47 & 52 & 47 & 112 & 142 & 621 & constante \\
\hline Oncometopiafacialis & 1 & 11 & 6 & 13 & 3 & 12 & 5 & 15 & 26 & 20 & 27 & 49 & 6 & 194 & constante \\
\hline Bahita infuscata & 16 & 33 & 0 & 7 & 18 & 11 & 7 & 2 & 15 & 26 & 53 & 89 & 142 & 419 & constante \\
\hline Docalidia bifurcata & 39 & 49 & 0 & 0 & 1 & 2 & 1 & 2 & 4 & 20 & 24 & 16 & 20 & 178 & constante \\
\hline Labocurtidia sp. & 347 & 334 & 0 & 0 & 3 & 10 & 2 & 3 & 9 & 111 & 83 & 112 & 382 & 1.396 & constante \\
\hline Joruma (Joruma) sp. & 11 & 3 & 8 & 13 & 31 & 6 & 0 & 0 & 2 & 31 & 0 & 60 & 22 & 187 & constante \\
\hline Dilobopterus costalimai & 2 & 9 & 0 & 0 & 0 & 2 & 1 & 10 & 16 & 6 & 22 & 15 & 5 & 88 & constante \\
\hline Coelidiana diminuta & 24 & 72 & 0 & 1 & 0 & 0 & 1 & 4 & 3 & 2 & 6 & 24 & 23 & 160 & constante \\
\hline Riqueza & 8 & 8 & 3 & 5 & 6 & 7 & 7 & 7 & 8 & 8 & 7 & 8 & 8 & & \\
\hline
\end{tabular}

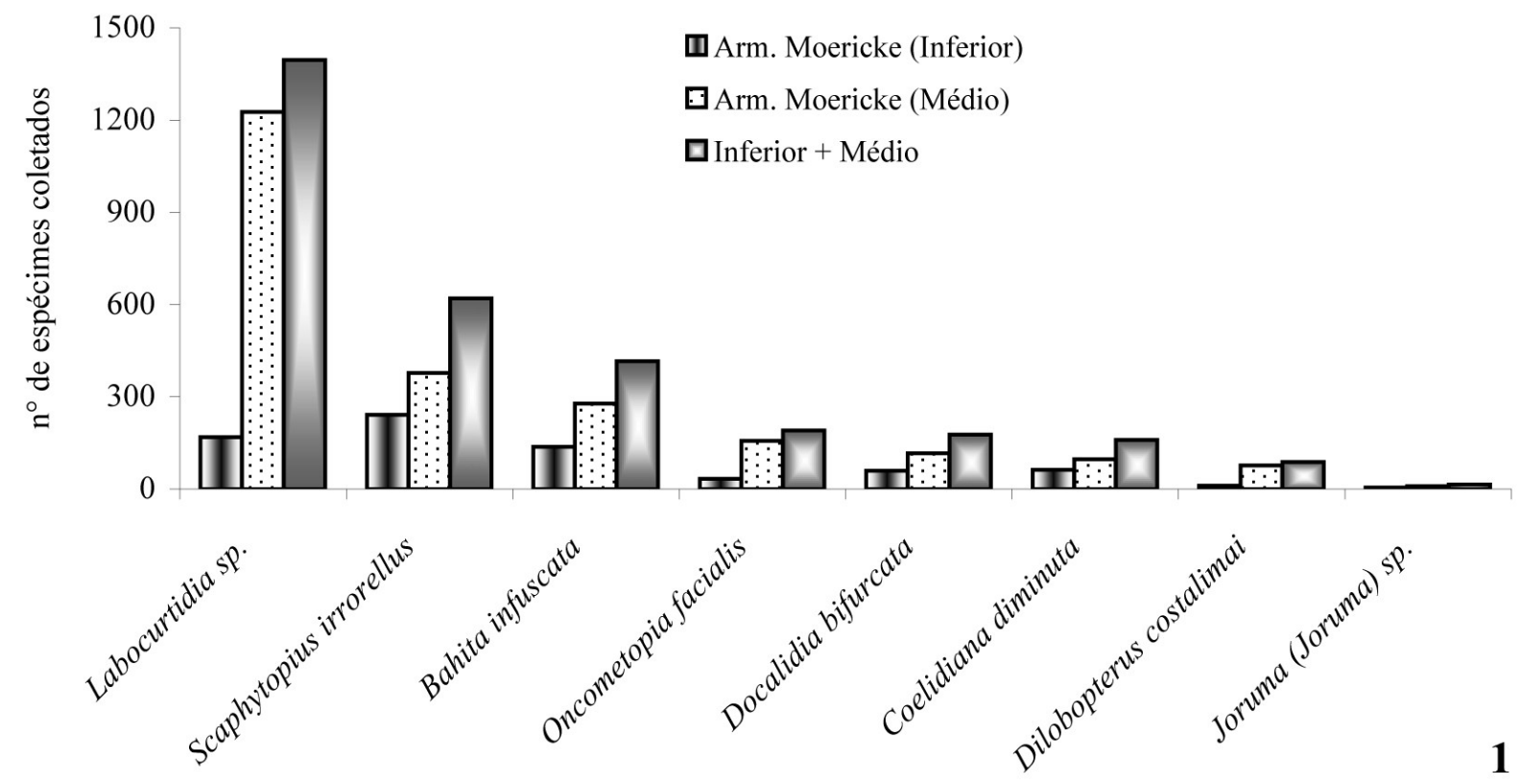

Fig. 1 - Espécies de cicadelídeos coletadas com armadilhas de Moericke instaladas nos terços médio e inferior das plantas de Coffea arabica no período de abril de 2005 a abril de 2006, em Cravinhos, SP, Brasil.

Deltocephalinae, segunda subfamília com maior número de espécimes coletados, foi representada por B. infuscata (Deltocephallini) e S. irrorellus (Scaphytopiini). Segundo Nielson (1985) apud YAMAMOto; Gravena (2000), mais de 50 espécies esta subfamília são vetoras de fitopatógenos. Na Costa Rica, Webi \& Godoy (1993) e Rojas et al. (2001) observaram que Scaphytopius Ball é um dos gêneros de Cicadellidae mais abundante em plantações de café. Segundo Rojas et al. (2001), Bahita sp. é uma espécie pouco freqüente em diferentes sistemas de cultivos de café.
A subfamília Cicadellinae, aqui representada por D. costalimai (Cicadellini) e O. facialis (Proconiini), foi a terceira mais abundante, tais espécies são importantes para a cultura do cafeeiro devido sua comprovada capacidadedetransmitirabactéria X. fastidiosa (ROBERTO et al., 1996; LOPES et al., 1996; KRÜGNER et al., 1998).

As subfamílias Neocoelidiinae e Typhlocybinae, representadas por C. diminuta (Neocoelidiini) e Joruma (Joruma)sp.(Jorumini), apresentarambaixafreqüênciade espécimescoletados.C. diminuta foirecentementedescrita a partir de exemplares coletados em uma plantaçãode caféem Bebedouro,SP(Chiamolera; CAVICHIOL, 2005). 


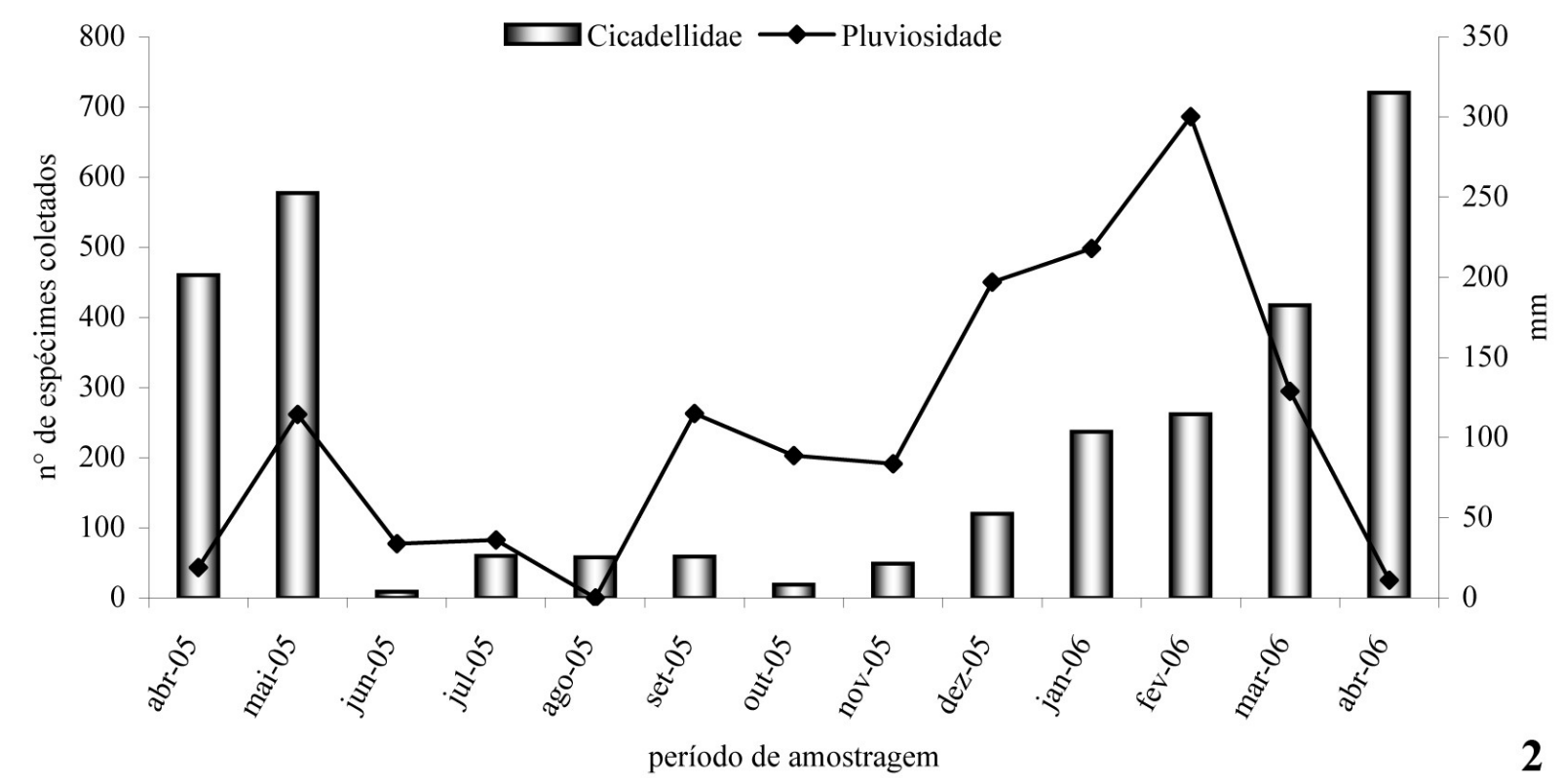

Fig. 2 - Variação mensal da pluviosidade ( $\mathrm{mm}$ ) e abundância de cicadelídeos coletados em cultivo de Coffea arabica no período de abril de 2005 a abril de 2006, em Cravinhos, SP, Brasil.

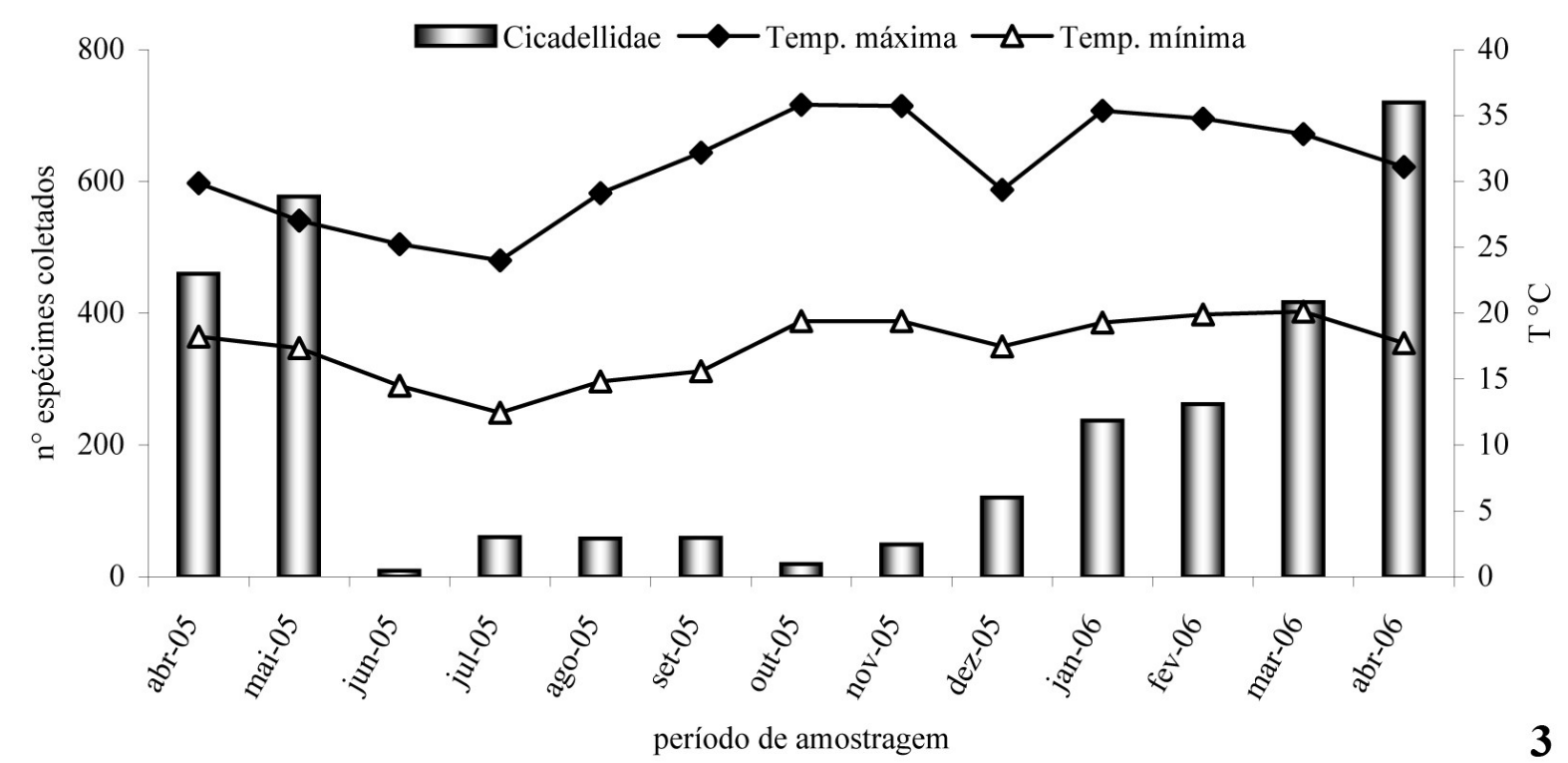

Fig. 3 - Variação mensal das temperaturas máxima e mínima $\left({ }^{\circ} \mathrm{C}\right)$ e abundância de cicadelídeos coletados em cultivo de Coffea arabica no período de abril de 2005 a abril de 2006, em Cravinhos, SP, Brasil.

Todas as espécies de cicadelídeos coletadas foram constantes no cafeeiro, com percentuais de ocorrência noslevantamentos quevariaram de $76,9 \%$ (D. costalimai e C. diminuta) a 100\% (S. irrorellus e O. facialis) (Tabela 2). Em junho, apenas $S$. irrorellus, O. facialis e Joruma (Joruma) sp. estiveram presentes no cafeeiro em baixa freqüência, talvez devido a uma aplicação de defensivo químico (thiamethoxam) para o controle de $L$. coffeella realizada durante o mês de maio; em outubro, foi efetuado ocontrole da mesma praga com defensivo químico (ditiocarbamato).

MeNEGUim et al. (2000) e LovATo et al. (2001) relataram que $O$. facialis e D. costalimai são espécies constantes em cafeeiros e viveiros de mudas no norte do Paraná e resultados semelhantes são relatados para o sul de Minas Gerais (SANTA-CECília et al., 2001). 
As amostragens realizadas com as armadilhas de Moericke (Fig. 1) instaladas na altura do terço médio das plantas de café foram responsáveis pela maior captura de cicadelídeos (2.474 exemplares $/ 76,3 \%$ do total de cicadelídeos coletados), enquanto as instaladas na altura do terço inferior das plantas coletaram apenas 769 exemplares $(23,7 \%)$. Para todas as espécies, mais de $60 \%$ dos exemplares coletados foram com as armadilhas de Moericke instaladas na altura do terço médio das plantas. As maiores diferenças observadas foram para as espécies Labocurtidia sp. (12,1\% no terço inferior das plantas $/ 87,9 \%$ no terço médio); $D$. costalimai $(12,5 \% / 87,5 \%)$ e O. facialis $(17,5 \% /$ $82,2 \%)$.

Em junho, início do período seco do ano, observou-se a redução no número de cigarrinhas coletadas. O aumento progressivo no número de insetos coletados ocorreu a partir de dezembro, no verão, período caracterizado pelo início da estação chuvosa e de temperaturas elevadas (Figs. 2 e 3). Lovato et al. (2001) e SANTA-CECILIA et al. (2001) constataram este mesmo padrão em cafeeiros do Paraná eMinas Gerais, respectivamente. Na cultura decitros, a população de cigarrinhas tende a aumentar a partir do início das chuvas e diminui significativamente no inverno e início da primavera (PAIVA et al., 1996), o que corrobora o padrão observado na cultura do café.

Dentre as espécies de cigarrinhas coletadas, apenas $D$. costalimai apresentou correlação positiva com a pluviosidade $(\mathrm{r}=0,31, \mathrm{p}=0,03)$; não houve correlação quando considerado o total de cicadelídeos coletados. Houve correlação positiva com a temperatura mínima para as espécies $D$. costalimai $(\mathrm{r}=0,37, \mathrm{p}$ $=0,007)$ e O. fascialis $(\mathrm{r}=0,28, \mathrm{p}=0,04)$; apenas $D$. costalimai apresentou correlação com a temperatura máxima $(\mathrm{r}=0,28, \mathrm{p}=0,04)$.

No sul de Minas Gerais, SANTA-CeCILIA et al. (2002) constataram aumento populacional de $O$. fascialis sob condições de temperatura e umidade relativa elevadas, com correlações significativas com a temperatura $(r=0,30)$ e com a umidade relativa $(r=0,45)$ e não com a pluviosidade.

\section{CONCLUSÕES}

No presente estudo constatou-se que os cicadelídeos associados à cultura do cafeeiro são mais abundantes no período chuvoso e que o terço médio das plantas é o local mais adequado para a instalação das armadilhas de Moericke visando à captura deste grupo de insetos. Labocurtidia sp. foi a espécie de cicadelídeo mais freqüente no cafeeiro.

\section{AGRADECIMENTOS}

Ao Prof. Dr. Gabriel Mejdalani eà MS.c. Raquel de Carvalho, do Departamento de Entomologia do Museu Nacional da Universidade Federal do Rio de Janeiro, pela identificação dos Cicadellidae e ao Sr. Edison Minohara, proprietário da Fazenda Palmares, pela cessão da área para realização deste estudo.

\section{REFERÊNCIAS}

ChiAmolera, L.D.B.; CAVICHIOLI, R.R. Notas sobre Coelidiana Oman (Hemiptera, Cicadellidae, Neocoelidiinae) e descrição de três novas espécies do Brasil. Revista Brasileira de Zoologia, v.22, n.2, p.494-500, 2005.

Godor, C.; Nielson, M.W. A review of the leafhoppergenus Jikradia with descriptions of four new species (Homoptera: Cicadellidae). Revista de Biología Tropical, v.46, n.3, p.739-748, 1998.

KRÜGNER, R.; LOPES, M.T.V.C.; SANTOS, J.S.; BERETTA, M.J.G.; LOPES, J.R.S. Transmission efficiency of Xylella fastidiosa by sharpshooters and identification of two new vector species. In: CONFERENCE OF THE INTERNACIONAL ORGANIZATION OF CITRUS VIROLOGISTS, 14., 1998, Campinas, SP. Anais. Campinas: 1998. p.81.

LOPES, J.R.S. Mecanismos de transmissão de Xylella fastidiosa por cigarrinhas. Laranja, v.17, n.1, p.79-92, 1996.

Lopes, J.R.S. Beretta, M.J.G.; HaraKaVa, R.; Almeida, R.R.; KRUgner R.; Garcia Junior, A.A. Confirmação da transmissão por cigarrinhas do agente causal da clorose variegada dos citros Xylella fastidiosa. Fitopatologia Brasileira, v.21, p.343, 1996. Suplemento.

Lovato, L.;SimÕes, H.C.;ZANDONÁ, C.;MEnEGUim, A.M.;Leite JúNIOR, R.P. Ocorrência de cigarrinhas vetoras da bactéria Xylella fastidiosa em lavouras cafeeiras no Estado do Paraná. In: SIMPÓSIO BRASILEIRO DE PESQUISA DOS CAFÉS DO BRASIL, 2., 2001, Vitória, ES. Anais. Vitória: 2001. p.2013-2021.

MenEGuim, A.M.; KimURA, L.A.; SimÕES, H.C. Levantamento da fauna de homópteros em viveiros de mudas de cafeeiro (Coffea arabica L.). In: SIMPÓSIO DE PESQUISADOSCAFÉSDOBRASIL, 1., 2000, Poços de Caldas, MG. Resumos expandidos. Poços deCaldas:2000.p.12631267.

Paiva, P.E.B.; Silva, J.L.; Gravena, S.; Yamamoto, P.T. Cigarrinhas de xilema em pomares de laranja do estado de São Paulo. Laranja, v.17, n.1, p.41-54, 1996.

Paradela Filho, O.; Sugimori, M.H.; Ribeiro, I.J.A.; Garcia Junior, A.; Beretta, M.J.G.; HaraKaWa, R.; Machado, M.A.;LARANJEIRA, F.F.; RODRIGUES NETO, J.; BERIAM, L.O.S. Constatação de Xylella fastidiosa em cafeeiro no Brasil. Summa Phytopathologica, v.23, n.1, p.46-49, 1997.

PerIOTO, N.W.; LARA, R.I.R.; SANTOS, J.C.C.; SILVA, T.C. DA Utilização de armadilhas de Moericke em ensaios de seletividade em himenópteros parasitóides. Arquivos do Instituto Biológico, São Paulo, v.67, p.93, 2000. Suplemento. Trabalho apresentado na REUNIÃO ANUAL DO INSTITUTO BIOLÓGICO, 13., 2000, São Paulo. Resumo 133. 
Queiroz-Voltan, R.B.; Paradela Filho, O.; Carelli, L.C. \& FAHL, J.I. Aspectos estruturais de cafeeiro infectado com Xylellafastidiosa. Bragantia, v.58,n.1, p.23-33,1998.

Rojas, L.; Godoy, C.; Hanson, P.; Hilje, L. A survey of homopteran species (Auchenorrhyncha) from coffee shrubs and poró and laurel trees in shaded coffee plantations, in Turrialba, Costa Rica. Revista de Biología Tropical, v.49, n.3/4, p.1057-1065, 2001.

Roberto, S.R., CoutinHO, A.; Lima, J.E.O.; Carlos, E.F. Transmissão de Xylella fastidiosa pelas cigarrinhas Dilobopterus costalimai, Acrogonia terminalis e Oncometopia facialis (Hemiptera: Cicadellidae) em citros. Fitopatologia Brasileira, v.21, n.4, p.517-518, 1996.

Santa-CeCília, L.V.C.; Gonçalves-Gervásio, R.C.R.; Souza, B.; Torres, A.F.; ReIS, P.R.; SouZA, J.C. DE Flutuação populacional e análise faunística de cigarrinhas que ocorrem em cafeeiros no sul do Estado de Minas Gerais. In: SIMPÓSIO BRASILEIRO DE PESQUISA DOS CAFÉS DO BRASIL, 2., 2001, Vitória, ES. Anais. Vitória: 2001. p.1951-1955.

SANTA-CECÍlIA, L.V.C.; GONÇALVES-GERVÁSIO, R.C.R.; SOUZA, B. Dinâmica populacional de Oncometopia facialis (Hemiptera: Cicadellidae) em cafeeiros. In: CON-
GRESSO BRASILEIRO DE PESQUISAS CAFEEIRAS, 28., 2002, Caxambu, MG. Trabalhos apresentados. Caxambu: 2002. p.363-364.

Silveira Neto, S.; Nakano, O.; Barbin, D.; Villa Nova, N.A. Manual de ecologia dos insetos. São Paulo: Agronômica Ceres, 1976. 419 p.

WebB, M.D.; Godoy, C. Review of the leafhopper tribe Scaphytopiini (Homoptera: Cicadellidae: Deltocephalinae) with a key to genera. Journal of Natural History, v.27, p.423-427, 1993.

Yамамото, P.T. Espécies eflutuação populacional de cigarrinhas e psilídeos (Hemiptera) em pomares cítricos. 1998. 112p. Tese (Doutorado em Agronomia) - Faculdade de Ciências Agrárias e Veterinárias, Universidade Estadual Paulista, Jaboticabal, 1998.

Yamamoto, P.T.; Gravena, S. Espécies e abundância de cigarrinhas e psilídeos (Homoptera) em pomares cítricos. Anais da Sociedade Entomológica do Brasil, Londrina, v.29, n.1, p.169-176, 2000.

Recebido em 20/10/06

Aceito em 14/9/07 